\title{
Identification of Hepatocellular Carcinoma-Related Potential Genes and Pathways Through Bioinformatic-Based Analyses
}

\author{
Zheng Wan, ${ }^{1,2}$ Xiaohong Zhang, ${ }^{1}$ Yuyang Luo, ${ }^{3}$ and Bin Zhao ${ }^{1,2}$
}

Background: Hepatocellular carcinoma (HCC) is one of the most prevalent tumors worldwide. However, the key roles of most molecules associated with tumor proliferation, invasion, and metastasis in HCC remain unclear. It is therefore important to explore potential mechanisms underlying tumorigenesis and to screen genes and pathways identified from such research for their role in pathogenesis.

Materials and Methods: We selected microarray data GSE62043 consisting of paired tissue samples from 100 HCC patients, then these data were analyzed to identify differentially expressed genes (DEGs). Next, gene ontology (GO) annotation and Kyoto Encyclopedia of Genes and Genomes (KEGG) pathway enrichment analyses were carried out to elucidate the biological processes, molecular function, cellular component (CC), and KEGG signaling pathways for the DEGs. We then constructed protein-protein interaction (PPI) networks, followed by a functional enrichment analysis, from which we obtained two significant gene modules. Finally, the gene expression data obtained from this test set were subjected to validation studies using an independent set of hepatocellular patient data archived in The Cancer Genome Atlas and Genotype-Tissue Expression (TCGA/GTEx) database.

Results: A total of 425 DEGs were identified that met both of our criteria for significance: (1) a $\mid \log 2$-fold change (FC) $\mid \geq 1.2$ and (2) an adjusted $p$ value $<0.01$. From these data, two significant gene modules, containing 28 pathway-related hub genes, were identified.

Conclusion: Through application of a test/validation algorithm using HCC datasets from two independent databases, we identified a number of genes that could serve as potential biomarkers for the molecular diagnosis and therapeutic intervention of HCC, including the known genes, IGF1, IGF2, NDC80,CDK1, CENPF, $C D C A 8, C C N B 1, B I R C 5, N C A P G$, and CDCA5, and the novel genes, CENPU and SPC25, which are associated with cell cycle, mitotic cell cycle, and organelle organization.

Keywords: differentially expressed genes, bioinformatics, hepatocellular carcinoma

\section{Introduction}

$\mathbf{H}$ EPATOCELlulaR CARCINOMA (HCC) is the most common form of primary liver cancer and is a leading cause of cancer-related deaths worldwide (Balogh et al., 2016). HCC occurs frequently in persons with chronic liver diseases such as cirrhosis caused by independent risk factors, including chronic hepatitis $\mathrm{B}$ virus or hepatitis $\mathrm{C}$ virus infection (Balogh et al., 2016).

An early diagnosis is essential for obtaining the best treatment results for $\mathrm{HCC}$ patients.

Patients suffering from late-stage HCC have a poor prognosis with limited therapeutic options. $\alpha$-Fetoprotein $(A F P)$, which is often expressed at high levels in HCC patients, has historically been used as a clinical biomarker (Chen et al., 1984). Currently, there is a substantial body of research concerning HCC-specific targeted therapies. These studies have mainly focused on the serine-threonine kinases, Raf-1 and B-Raf, and the receptor tyrosine kinase activity of vascular endothelial growth factor receptors (VEGFR-1,VEGFR-2, and $V E G F R-3)$ and platelet-derived growth factor receptor $\beta$ (PDGFR- $\beta$ ) (Wilhelm et al., 2004; Chang et al., 2007). Although the prognosis for metastatic or unresectable HCC has improved in recent years due to the approval of the target drug, Sorafenib (Llovet et al., 2008), the overall survival rate is still poor. This suggests that the mechanisms underlying HCC

\footnotetext{
${ }^{1}$ School of Medicine, Xiamen University, Xiamen, China.

${ }^{2}$ Department of Oncology and Vascular Interventional Radiology, Zhongshan Hospital Affiliated to Xiamen University, Xiamen, China.

${ }^{3}$ ShenZhen College of International Education, Shenzhen, China.
} 
progression require further exploration, which would hopefully lead to the discovery of novel therapeutic targets.

Gene chip microarrays can provide genome-wide expression profiles (Lee et al., 2013) that can be analyzed using unbiased bioinformatic tools to discover new therapeutic targets. Exporting data from these analyses into modern pathway profiling software could uncover meaningful clues toward novel understanding (Guo et al., 2017). Vast datasets have been developed from HCC patient specimens in recent years (Wang et al., 2015; Mou et al., 2017), and a lot of differentially expressed genes (DEGs) have been identified and characterized using gene ontology (GO) and signaling pathway analyses (Zhao et al., 2019).

In this study, we downloaded the GSE62043 mRNA expression profiles from the website of Gene Expression Omnibus (GEO). GEO is a public online database for uploading, archiving, retrieving, and querying microarray datasets. DEG analyses were carried out between primary tumor tissues (marked tumor) of HCC patients and paired, adjacent nonmalignant tissues (marked control) with $\mathrm{R}$ software (Dessau and Pipper, 2008) using the built-in limma et al. packages (Smyth, 2005). Then, functional and signaling pathway enrichment analyses were performed with the online bioinformatics resource DAVID. We also constructed protein-protein interaction (PPI) networks of the DEGs and identified those genes that appeared in two or more analyses (module genes). Finally, we validated these candidate module genes using separate datasets available through The Cancer Genome Atlas and Genotype-Tissue Expression (TCGA/GTEx) databases. Using this approach, we identified several potentially important HCC-related genes and pathways for further study to help define their roles in diagnosis, prognosis, and therapeutic treatment.

Table 1. Four Hundred Twenty-Five Differentially Expressed Genes Were Identified In GSE62043, InCluding 48 Upregulated Genes and 377 Downregulated Genes IN 100 Paired SAMPLES OF HCC PATIENTS

\begin{tabular}{|c|c|}
\hline$D E G s$ & Gene name \\
\hline $\begin{array}{l}\text { Upregulated } \\
\text { genes }\end{array}$ & $\begin{array}{l}\text { SPINK1, CENPF, BIRC5, NCAPG, MDK, CCNB1, CDKN3, SQLE, TK1, OIP5, UBE2T, NDC80, } \\
\text { CDCA5, CDCA8, FAM83D, KIAAO101, STMN1, SPC25, COX7B2, TOP2A,CDKN2A,CDT1,CDK1, } \\
\text { GNAZ, MAGEA2B, ESM1, IGF2BP3, EZH2, HOXA13,CAP2, AKR1B1, PRC1, ENAH, TYMS, } \\
\text { CENPU, MND1, CDCA2, LCN2, SPON2, CGREF1, APLN, SULT1C2, MAGEA12, SMPX, CDC6, } \\
\text { CEP55, CLGN, AKR1C3 }\end{array}$ \\
\hline $\begin{array}{l}\text { Downregulated } \\
\quad \text { genes }\end{array}$ & 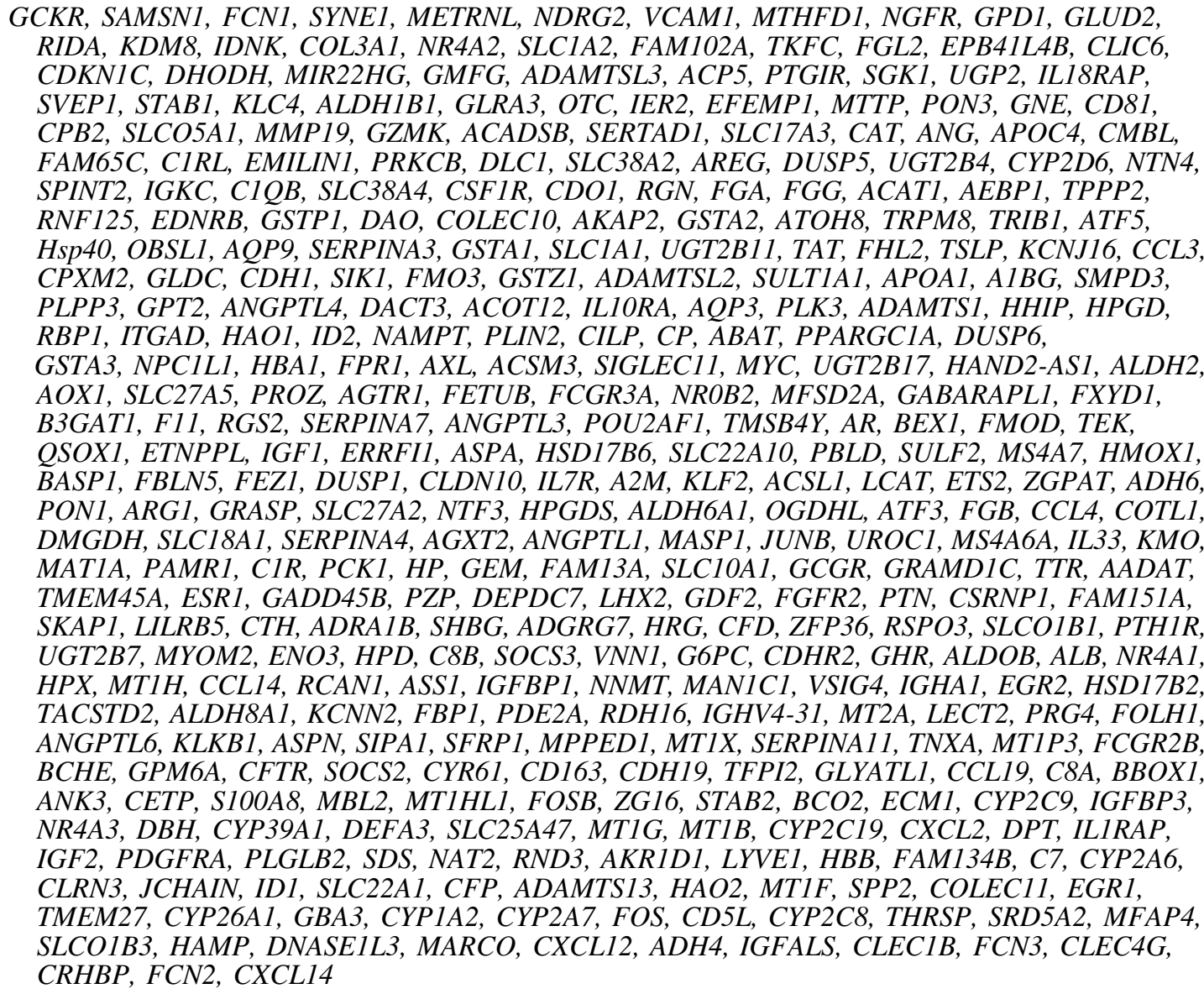 \\
\hline
\end{tabular}

The differentially expressed genes are listed from the smallest to the largest adjusted $p$ value.

DEG, differentially expressed gene; HCC, hepatocellular carcinoma. 
FIG. 1. GO terms and KEGG pathways of DEGs significantly enriched in HCC. DEG, differentially expressed gene; GO, gene ontology; HCC, hepatocellular carcinoma; KEGG, Kyoto Encyclopedia of Genes and Genomes.

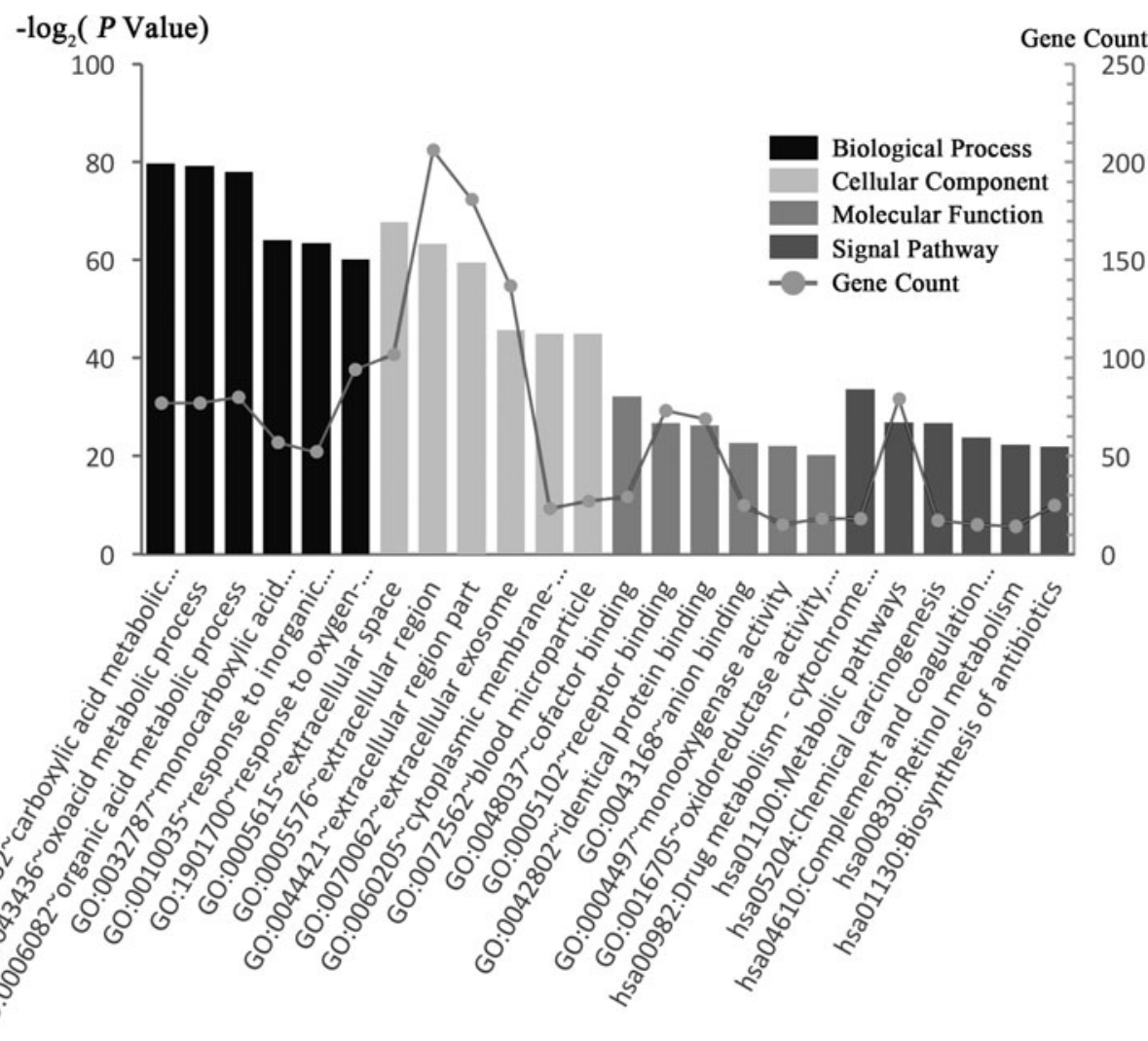

\section{Materials and Methods}

\section{Microarray data and data preprocessing}

Data from the Agilent microarray file GSE62043 (Thurnherr et al., 2016) were downloaded from the public NCBIGEO database (Edgar et al., 2008; Barrett et al., 2009) and executed on the GPL6480 platform. GSE62043 contains 100 paired primary and adjacent nonmalignant tissue samples from HCC patients. After the normalized $\log 2$ ratio (Cy5/ Cy3) representing tumor/normal of the GSE62043 dataset, normalized by the loess and quantile method (Smyth and Speed, 2003), was downloaded, probe identification numbers were converted to gene symbols using as a reference the Whole Human Genome Microarray $4 \times 44 \mathrm{~K}$ G4112F (Probe Name Version). When multiple probes corresponded to the same gene, the probe with the greatest $p$ value from the downstream differential analysis was chosen to determine the differential gene expression value.

\section{Identification of DEGs}

DEG analysis refers to identification of genes that are identified as being expressed at significantly different levels by multiple modes of analysis (Korpelainen and Tuimala, 2014). We used limma (Smyth, 2005) and R (Dessau and Pipper, 2008) packages to identify the DEGs in primary tumor tissues (marked tumor) of HCC patients and compared them with adjacent nonmalignant tissues (marked normal) of HCC patients. Genes with | log2-fold change $(\mathrm{FC}) \mid \geq 1.2$ and adjusted $p$ values $<0.01$ (moderated $t$ statistics, corrected by Benjamini and Hochberg's method) were used in the next analysis stage (Benjamini and Hochberg, 1995).

\section{GO enrichment and pathway analysis}

The GO (Ashburner et al., 2000) is a structured vocabulary of terms describing gene products according to the biological process (BP), molecular function (MF), and cellular component (CC). The Kyoto Encyclopedia of Genes and Genomes (KEGG) (Ogata et al., 1999) provides data resources of known biological metabolic pathways. We used DAVID (Dennis et al., 2003), a web-accessible program that integrates functional genomic annotations with intuitive graphical summaries, to view the GO and KEGG enrichment of DEGs; a $p$ value $<0.05$ was considered statistically significant.

\section{PPI networks and module analysis}

The Search Tool for the Retrieval of Interacting Genes (STRING, Version 11.0) (Franceschin et al., 2013) database was used to retrieve DEGs' encoded protein and PPI network information. This database contains $>24.6$ million proteins and 2 billion interactions involved in 5,090 organisms. We uploaded the DEGs into the STRING database and set the interaction score $\geq 0.900$ (highest confidence) as the significant threshold. Then, the PPI networks were constructed using Cytoscape (Kohl et al., 2011; Smoot et al., 2011) software. The Molecular Complex Detection (MCODE) built in Cytoscape is an automated method that was used to analyze highly interconnected modules as molecular complexes or clusters. The analysis parameters were set by default. The functional enrichment analysis was executed for DEGs, from 
Table 2. The Top Five Pathways in GO and KEGG Enrichment Analysis of DEGs

\begin{tabular}{|c|c|c|c|}
\hline Category & Term & Count & p Value \\
\hline \multicolumn{4}{|l|}{ Upregulated DEGs } \\
\hline GOTERM_BP_FAT & GO:1903047 mitotic cell cycle process & 22 & $3.03 \mathrm{E}-15$ \\
\hline GOTERM_BP_FAT & GO:0051301 cell division & 19 & $4.31 \mathrm{E}-15$ \\
\hline GOTERM_BP_FAT & GO:0007059 chromosome segregation & 16 & $7.26 \mathrm{E}-15$ \\
\hline GOTERM_BP_FAT & GO:0022402 cell cycle process & 25 & $8.33 \mathrm{E}-15$ \\
\hline GOTERM_BP_FAT & GO:0000278 mitotic cell cycle & 22 & $1.59 \mathrm{E}-14$ \\
\hline GOTERM_CC_FAT & GO:0000775 chromosome, centromeric region & 9 & $1.01 \mathrm{E}-07$ \\
\hline GOTERM_CC_FAT & GO:0000793 condensed chromosome & 9 & $2.27 \mathrm{E}-07$ \\
\hline GOTERM_CC_FAT & GO:0005694 chromosome & 15 & 4.96E-07 \\
\hline GOTERM_CC_FAT & GO:0098687 chromosomal region & 10 & $9.25 \mathrm{E}-07$ \\
\hline GOTERM_CC_FAT & GO:0044427 chromosomal part & 13 & $5.38 \mathrm{E}-06$ \\
\hline GOTERM_MF_FAT & GO:0003682 chromatin binding & 7 & 0.001513775 \\
\hline GOTERM_MF_FAT & GO:0019899 enzyme binding & 11 & 0.01131879 \\
\hline GOTERM_MF_FAT & GO:0044877 macromolecular complex binding & 9 & 0.014605682 \\
\hline GOTERM_MF_FAT & GO:0019900 kinase binding & 6 & 0.016144185 \\
\hline GOTERM_MF_FAT & GO:0042802 identical protein binding & 9 & 0.018143497 \\
\hline KEGG_PATHW'AY & hsa04110: Cell cycle & 4 & 0.002696712 \\
\hline KEGG_PATHWAY & hsa04115: p53 signaling pathway & 3 & 0.010269564 \\
\hline KEGG_PATHWAY & hsa05206: MicroRNAs in cancer & 4 & 0.026640176 \\
\hline \multicolumn{4}{|l|}{ Downregulated DEGs } \\
\hline GOTERM_BP_FAT & GO:0019752 carboxylic acid metabolic process & 76 & $1.24 \mathrm{E}-27$ \\
\hline GOTERM_BP_FAT & GO:0043436 oxoacid metabolic process & 76 & $1.82 \mathrm{E}-27$ \\
\hline GOTERM_BP_FAT & GO:0006082 organic acid metabolic process & 79 & $3.25 \mathrm{E}-27$ \\
\hline GOTERM_BP_FAT & GO:0032787 monocarboxylic acid metabolic process & 56 & $6.95 \mathrm{E}-22$ \\
\hline GOTERM_BP_FAT & GO:0009605 response to external stimulus & 105 & $4.94 \mathrm{E}-18$ \\
\hline GOTERM_CC_FAT & GO:0005615 extracellular space & 97 & $8.91 \mathrm{E}-23$ \\
\hline GOTERM_CC_FAT & GO:0005576 extracellular region & 191 & $7.67 \mathrm{E}-22$ \\
\hline GOTERM_CC_FAT & GO:0044421 extracellular region part & 169 & $8.99 \mathrm{E}-21$ \\
\hline GOTERM_CC_FAT & GO:0070062 extracellular exosome & 128 & 8.30E-16 \\
\hline GOTERM_CC_FAT & GO:1903561 extracellular vesicle & 128 & $1.18 \mathrm{E}-15$ \\
\hline GOTERM_MF_FAT & GO:0048037 cofactor binding & 26 & 2.09E-09 \\
\hline GOTERM_MF_FAT & GO:0005102 receptor binding & 68 & $6.11 \mathrm{E}-09$ \\
\hline GOTERM_MF_FAT & GO:0043168 anion binding & 25 & $1.98 \mathrm{E}-08$ \\
\hline GOTERM_MF_FAT & GO:0042802 identical protein binding & 60 & 3.67E-07 \\
\hline GOTERM_MF_FAT & GO:0031406 carboxylic acid binding & 17 & $7.52 \mathrm{E}-07$ \\
\hline KEGG_PATHWAY & hsa00982: Drug metabolism - cytochrome P450 & 18 & 2.36E-11 \\
\hline KEGG_PATHWAY & hsa05204: Chemical carcinogenesis & 17 & $3.26 \mathrm{E}-09$ \\
\hline KEGG_PATHWAY & hsa04610: Complement and coagulation cascades & 15 & $2.67 \mathrm{E}-08$ \\
\hline KEGG_PATHWAY & hsa01100: Metabolic pathways & 73 & $4.06 \mathrm{E}-08$ \\
\hline KEGG_PATHWAY & hsa00830: Retinol metabolism & 14 & $8.41 \mathrm{E}-08$ \\
\hline
\end{tabular}

GO, gene ontology; KEGG, Kyoto Encyclopedia of Genes and Genomes.

which two significant modules of genes were identified with $p<0.05$ set as the threshold.

\section{Validation of gene expression and overall survival of module genes}

Gene Expression Profiling Interactive Analysis (Tang et al., 2017) is a web sever, which contains the TCGA and GTEx datasets. First, we uploaded the genes identified by the PPI networks to validate the consistency of gene expression between the GSE62043 and TCGA/GTEx liver hepatocellular carcinoma (LIHC) datasets; we set the parameters, $|\log 2 \mathrm{FC}|$ cutoff: 1.2 and $p$ value cutoff: 0.01 , as the threshold criteria. Next, we performed the overall survival analysis. The patients in the TCGA/GTEx dataset were divided into high and low expression groups using the median transcripts per kilobase million (TPM) as a breakpoint, and significance was determined using a log-rank test with $p<0.05$. The functional enrichment analysis was executed to annotate the final potential key genes with the filter parameter, $p$ value $<1 \times 10^{-10}$, as the threshold in the GenCLiP 2.0 website (Wang et al., 2014).

\section{Results}

\section{Identification of DEGs}

In this study, we identified DEGs from 100 paired primary tumor tissues (tumor) using limma packages, compared with adjacent nonmalignant tissues (control). Using both $\mid \log 2-$ fold change (FC) $\mid \geq 1.2$ and adjusted $p$ value $<0.01$ criteria, a total of 425 genes were identified, including 48 upregulated genes and 377 downregulated genes (Table 1).

\section{GO enrichment and pathway analysis}

To further explore potential targets of these DEGs in HCC, we performed GO and pathway analyses on HCC with the 


\section{A}

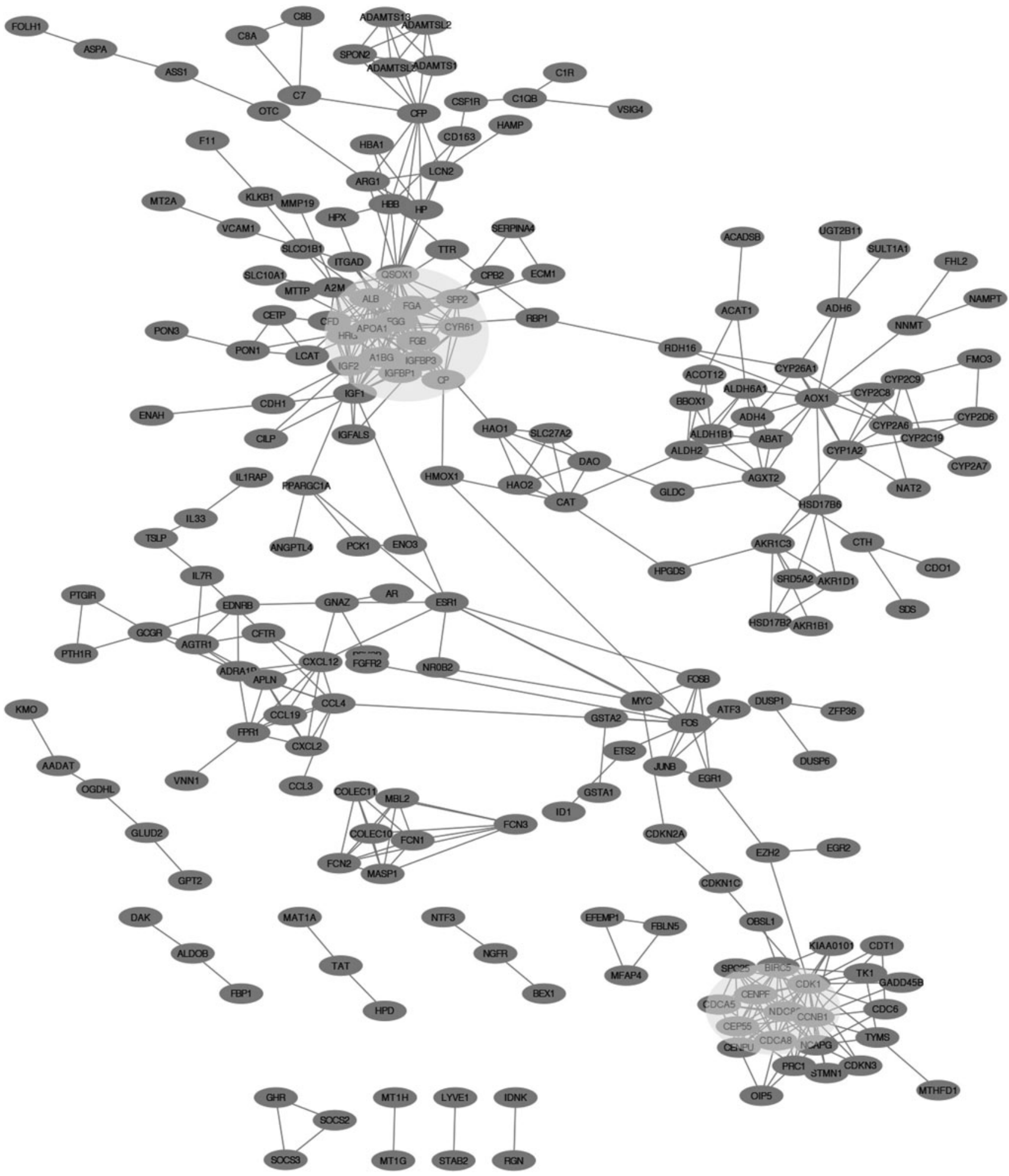

FIG. 2. PPI networks of DEGs. (A) Based on the STRING online database, 211 genes/nodes were filtered into the DEG PPI network. The two highlighted circle areas are the most significant modules. (B) The most significant module 1 from the PPI network (all genes are downregulated genes and labeled light gray). (C) The second significant module 2 from the PPI network (all genes are upregulated genes and labeled dark gray). PPI, protein-protein interaction. 

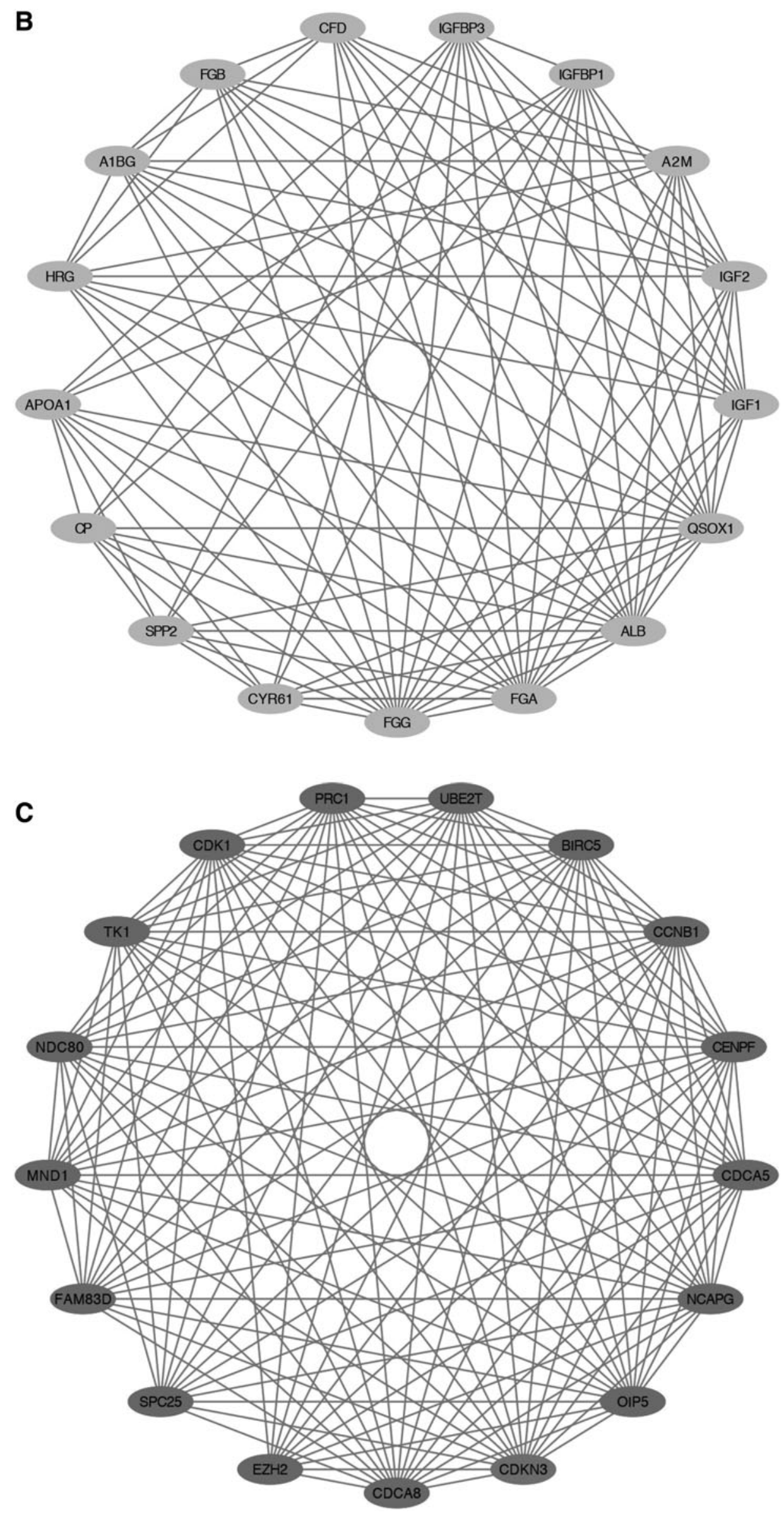

FIG. 2. (Continued). 
Table 3. Functional and Pathway Enrichment of Module 1 Genes

\begin{tabular}{|c|c|c|c|c|}
\hline Category & Term & Count & $\mathrm{p}$ Value & Genes \\
\hline GOTERM_BP_FAT & $\begin{array}{l}\text { GO:0002576 platelet } \\
\text { degranulation }\end{array}$ & 13 & 4.67E-24 & $\begin{array}{c}A 2 M, C F D, Q S O X 1, F G B, I G F 1, F G G, I G F 2, \\
A P O A 1, A L B, H R G, F G A, A 1 B G, S P P 2\end{array}$ \\
\hline GOTERM_BP_FAT & $\begin{array}{l}\text { GO: } 0045055 \sim \text { regulated } \\
\text { exocytosis }\end{array}$ & 13 & $9.72 \mathrm{E}-19$ & $\begin{array}{c}A 2 M, C F D, Q S O X 1, F G B, I G F 1, F G G, I G F 2, \\
A P O A 1, A L B, H R G, F G A, A 1 B G, S P P 2\end{array}$ \\
\hline GOTERM_BP_FAT & GO:0006887 exocytosis & 13 & $5.87 \mathrm{E}-17$ & $\begin{array}{c}A 2 M, C F D, Q S O X 1, F G B, I G F 1, F G G, I G F 2, \\
A P O A 1, A L B, H R G, F G A, A 1 B G, S P P 2\end{array}$ \\
\hline GOTERM_CC_FAT & $\begin{array}{l}\text { GO:0034774 secretory } \\
\text { granule lumen }\end{array}$ & 13 & $1.49 \mathrm{E}-24$ & $\begin{array}{c}A 2 M, C F D, Q S O X 1, F G B, I G F 1, F G G, I G F 2, \\
A P O A 1, A L B, H R G, F G A, A 1 B G, S P P 2\end{array}$ \\
\hline GOTERM_CC_FAT & $\begin{array}{l}\text { GO:0060205 cytoplasmic } \\
\text { membrane-bounded vesicle } \\
\text { lumen }\end{array}$ & 13 & $1.89 \mathrm{E}-23$ & $\begin{array}{l}A 2 M, C F D, Q S O X 1, F G B, I G F 1, F G G, I G F 2, \\
\quad A P O A 1, A L B, H R G, F G A, A 1 B G, S P P 2\end{array}$ \\
\hline GOTERM_CC_FAT & GO:0031983 vesicle lumen & 13 & $2.13 \mathrm{E}-23$ & $\begin{array}{c}A 2 M, C F D, Q S O X 1, F G B, I G F 1, F G G, I G F 2, \\
A P O A 1, A L B, H R G, F G A, A 1 B G, S P P 2\end{array}$ \\
\hline GOTERM_MF_FAT & $\begin{array}{l}\text { GO:0005102 receptor } \\
\text { binding }\end{array}$ & 10 & $1.73 \mathrm{E}-06$ & $\begin{array}{l}A 2 M, F G B, I G F 1, F G G, I G F 2, A P O A 1, H R G, \\
\quad F G A, I G F B P 1, C Y R 61\end{array}$ \\
\hline GOTERM_MF_FAT & $\begin{array}{l}\text { GO:0019838 growth factor } \\
\text { binding }\end{array}$ & 4 & $2.34 \mathrm{E}-04$ & $A 2 M, I G F B P 3, I G F B P 1, C Y R 61$ \\
\hline GOTERM_MF_FAT & $\begin{array}{l}\text { GO:0005520 insulin-like } \\
\text { growth factor binding }\end{array}$ & 3 & $3.27 \mathrm{E}-04$ & IGFBP3, IGFBP1, CYR61 \\
\hline KEGG_PATHWAY & $\begin{array}{l}\text { hsa04610: Complement and } \\
\text { coagulation cascades }\end{array}$ & 5 & $1.12 \mathrm{E}-06$ & $A 2 M, C F D, F G B, F G G, F G A$ \\
\hline KEGG_PATHWAY & hsa04611: Platelet activation & 3 & 0.011696553 & $F G B, F G G, F G A$ \\
\hline KEGG_PATHWAY & $\begin{array}{l}\text { hsa05150: Staphylococcus } \\
\text { aureus infection }\end{array}$ & 2 & 0.068510167 & $C F D, F G G$ \\
\hline
\end{tabular}

criterion of $p$ value $<0.05$ (Fig. 1). As shown in Figure 1, it shows the top six significant terms for each of the following: the BP, CC, MF, and KEGG pathways of DEGs, respectively.

We also show annotation of the upregulated genes as well as downregulated genes. As shown in Table 2, in the BP group, the upregulated DEGs were mainly enriched for genes in- volved in the mitotic/cell cycle processes, cell division, and chromosome segregation. The downregulated DEGs were enriched genes in the carboxylic acid metabolic processes, oxoacid metabolic process, and other organic acid metabolic processes. In the CC group, the upregulated DEGs were mainly enriched for genes associated with the chromosome,

Table 4. Functional and Pathway Enrichment of Module 2 Genes

\begin{tabular}{|c|c|c|c|c|}
\hline Category & Term & Count & $\mathrm{p}$ Value & Genes \\
\hline GOTERM_BP_FAT & $\begin{array}{l}\text { GO:0000819 } \sim \text { sister } \\
\text { chromatid segregation }\end{array}$ & 10 & $1.21 \mathrm{E}-16$ & $\begin{array}{l}\text { CDCA5, CEP55, BIRC5, CCNB1, NCAPG, } \\
\text { CENPU, CDCA8, CENPF, NDC80, SPC } 25\end{array}$ \\
\hline GOTERM_BP_FAT & $\begin{array}{l}\text { GO: } 0098813 \sim \text { nuclear } \\
\quad \text { chromosome segregation }\end{array}$ & 10 & $1.17 \mathrm{E}-15$ & $\begin{array}{l}\text { CDCA5, CEP55, BIRC5, CCNB1, NCAPG, } \\
\text { CENPU, CDCA8, CENPF, NDC80, SPC } 25\end{array}$ \\
\hline GOTERM_BP_FAT & $\begin{array}{l}\text { GO:0007059 chromosome } \\
\text { segregation }\end{array}$ & 10 & $4.64 \mathrm{E}-15$ & $\begin{array}{l}\text { CDCA5, CEP55, BIRC5, CCNB1, NCAPG, } \\
\text { CENPU, CDCA8, CENPF, NDC80, SPC } 25\end{array}$ \\
\hline GOTERM_CC_FAT & $\begin{array}{l}\mathrm{GO}: 0098687 \sim \text { chromosomal } \\
\text { region }\end{array}$ & 9 & 4.13E-12 & $\begin{array}{l}\text { CDCA5, BIRC5, CCNB1, CDK1, CENPU, } \\
\text { CDCA8, CENPF, NDC80, SPC } 25\end{array}$ \\
\hline GOTERM_CC_FAT & $\begin{array}{l}\text { GO: } 0000775 \sim \text { chromosome } \\
\text { centromeric region }\end{array}$ & 8 & $6.56 \mathrm{E}-12$ & $\begin{array}{l}\text { CDCA5, BIRC5, CCNB1, CENPU, CDCA8, } \\
\text { CENPF, NDC80, SPC } 25\end{array}$ \\
\hline GOTERM_CC_FAT & $\begin{array}{l}\text { GO:0000793 condensed } \\
\text { chromosome }\end{array}$ & 8 & $1.38 \mathrm{E}-11$ & $\begin{array}{l}\text { CDCA5, BIRC5, CCNB1, NCAPG, CENPU, } \\
\text { CENPF, NDC80, SPC } 25\end{array}$ \\
\hline GOTERM_MF_FAT & $\begin{array}{l}\text { GO:0035173 histone kinase } \\
\text { activity }\end{array}$ & 2 & 0.006123483 & $C C N B 1, C D K 1$ \\
\hline GOTERM_MF_FAT & $\begin{array}{l}\text { GO:0003682 chromatin } \\
\text { binding }\end{array}$ & 3 & 0.009912687 & $C D C A 5, C D K 1, C E N P F$ \\
\hline GOTERM_MF_FAT & $\begin{array}{l}\text { GO:0004693 cyclin- } \\
\text { dependent protein } \\
\text { serine/threonine kinase } \\
\text { activity }\end{array}$ & 2 & 0.010936591 & $C C N B 1, C D K 1$ \\
\hline KEGG_PATHWAY & $\begin{array}{l}\text { hsa04115: p53 signaling } \\
\text { pathway }\end{array}$ & 2 & 0.028939863 & $C C N B 1, C D K 1$ \\
\hline KEGG_PATHWAY & $\begin{array}{l}\text { hsa04914: Progesterone- } \\
\text { mediated oocyte maturation }\end{array}$ & 2 & 0.037469108 & $C C N B 1, C D K 1$ \\
\hline KEGG_PATHWAY & hsa04114: Oocyte meiosis & 2 & 0.047638096 & $C C N B 1, C D K 1$ \\
\hline
\end{tabular}


Table 5. VAlidation of the Gene Expression Changes of Two Modules Between GSE62043 AND TCGA/GTEx

\begin{tabular}{|c|c|c|}
\hline Gene & $\begin{array}{c}\text { HCC patients } \\
\text { (GSE62043) }\end{array}$ & $\begin{array}{l}\text { LIHC patients } \\
\text { (TCGA/GTEx) }\end{array}$ \\
\hline \multicolumn{3}{|l|}{ Module 1} \\
\hline IGFBP3 & Down & Down \\
\hline$I G F B P 1$ & Down & - \\
\hline$A 2 M$ & Down & - \\
\hline$I G F 2$ & Down & Down \\
\hline$I G F 1$ & Down & Down \\
\hline QSOX1 & Down & - \\
\hline$\widetilde{A} L B$ & Down & - \\
\hline$F G A$ & Down & - \\
\hline$F G G$ & Down & - \\
\hline CYR61 & Down & Down \\
\hline$S P P 2$ & Down & - \\
\hline$C P$ & Down & - \\
\hline$A P O A 1$ & Down & - \\
\hline$H R G$ & Down & - \\
\hline$A 1 B G$ & Down & - \\
\hline$F G B$ & Down & - \\
\hline$C F D$ & Down & - \\
\hline \multicolumn{3}{|l|}{ Module 2} \\
\hline NDC80 & Up & Up \\
\hline$C D K 1$ & $\mathrm{Up}$ & Up \\
\hline$C E N P F$ & Up & Up \\
\hline CDCA 8 & Up & Up \\
\hline$C C N B 1$ & Up & Up \\
\hline BIRC5 & Up & Up \\
\hline CEP55 & Up & - \\
\hline$N C A P G$ & Up & Up \\
\hline$S P C 25$ & Up & Up \\
\hline$C D C A 5$ & Up & Up \\
\hline$C E N P U$ & Up & Up \\
\hline
\end{tabular}

The short horizontal line indicates that the expression of the gene does not meet the cutoff: $|\log 2 \mathrm{FC}| \geq 1.2$ and $p$ value $<0.01$ in LIHC. LIHC, liver hepatocellular carcinoma.

centromeric regions, and condensed chromosome. The downregulated DEGs were enriched for genes associated with the extracellular space, extracellular region, and extracellular region part. In the MF group, the upregulated DEGs were mainly enriched from genes associated with chromatin binding, enzyme binding, and macromolecular complex binding; and the downregulated DEGs were enriched for genes encoding proteins for cofactor binding, receptor binding, and anion binding. In the KEGG pathway group, the upregulated DEGs were enriched for genes in the cell cycle, signaling pathway, and microRNAs in cancer; and the downregulated DEGs were enriched for those associated with drug metabolism, including cytochrome P450s, chemical carcinogenesis, and the complement and coagulation cascades.

\section{PPI networks and module analysis}

All of the DEGs were uploaded onto the STRING website and were analyzed with Cytoscape software. A total of 211 nodes with 475 edges with scores $>0.900$ (highest confidence) were selected to construct the PPI networks (Fig. 2). Two significant modules were selected using the MCODE plug-in. Genes in module 1 are downregulated, while the genes in module 2 are upregulated (Fig. 2B, C). Module 1 consisted of 17 nodes/genes and 99 edges (Fig. 2B), which are mainly associated with platelet degranulation (BP), secretory granule lumen $(\mathrm{CC})$, receptor binding $(\mathrm{MF})$, and complement and coagulation cascades (KEGG) (Table 3). Module 2 consisted of 11 nodes/genes and 49 edges (Fig. 2C), which are mainly associated with sister chromatid segregation (BP), chromosomal region (CC), histone kinase activity (MF), and the p53 signaling pathway (KEGG) (Table 4).

\section{Validation of gene expression and overall survival of module genes}

To assess whether gene expression changes are consistent between specimens from the test HCC (GSE62043) and validation LIHC (TCGA/GTEx) datasets, we validated these two module genes (total 28 genes) in the GEPIA website. We found that $C Y R 61, I G F 1, I G F 2$, and IGFBP3 in module 1 were downregulated in tumor versus normal in the LIHC datasets (built in TGCA/GTEx), which is in accordance with GSE62043 HCC samples (Table 5). The genes of module 2, including NDC80, CDK1, CENPF, CDCA8, CCNB1, BIRC5, $N C A P G, S P C 25, C D C A 5$, and $C E N P U$, were upregulated in tumor versus normal in the LIHC datasets, which is in accordance with GSE62043 HCC patients (Table 5).

We picked out those 14 genes with the criterion: the trend of gene expression in GSE62043 is consistent with LIHC datasets built in TCGA/GTEx. For further verification, overall survival of each of the above 14 candidate genes was calculated. As shown in Figure 3A and B, the downregulated genes (including $I G F 1$ and $I G F 2$ ) have lower percent survival in the low expression group compared with the high expression group as well as the other upregulated genes (including NDC80, CDK1, CENPF, CDCA8, CCNB1, $B I R C 5, N C A P G, S P C 25, C D C A 5$, and $C E N P U)$. The reason we discarded $C Y R 61$ and IGFBP3 is that the log-rank $p$ value of CYR61 did not meet standard criteria and the percent survival of IGFBP3 is opposite to the other downregulated genes (IGF1 and IGF2) (Supplementary Fig. S1). All retained candidate genes were involved in cell cycle, mitotic cell cycle, and organelle organization pathways (Fig. 4).

\section{Discussion}

This study was designed to identify potential HCC-related genes by comparing tumor tissues with adjacent nonmalignant tissues of patients suffering from HCC. Forty-eight upregulated and 377 downregulated DEGs were identified. Then, we performed GO and KEGG annotation analyses for DEGs. Subsequently, a DEG PPI network was constructed and 211 nodes/genes were identified with 475 edges, and two most significant modules were chosen from the PPIs, of which 28 central node genes were selected for further validation of gene expression and overall survival aspects in the TCGA/GTEx database. Finally, 12 genes, IGF1, IGF2, NDC80, CDK1, CENPF, CDCA8, CCNB1, BIRC5, NCAPG, $S P C 25, C D C A 5$, and $C E N P U$, which were significantly associated with cell cycle, mitotic cell cycle, and organelle organization, were identified.

Insulin-like growth factor $1(I G F 1)$ acts in response to growth hormone. It plays an important role in childhood growth and continues to have anabolic effects in adults (Keating, 2008). Wang et al. (2017) demonstrated that the serum IGF1 level is predictive of progression and survival in 
A

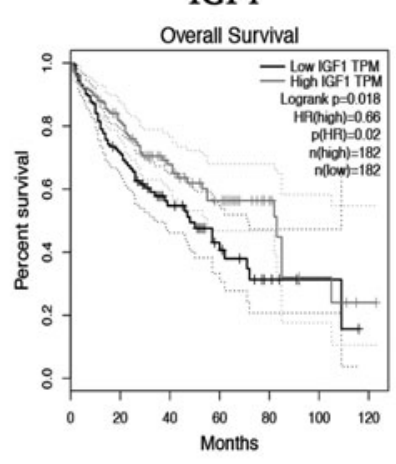

B $\quad \mathrm{NDC80}$

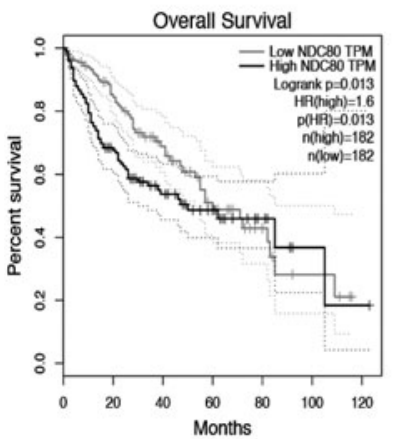

CCNB1

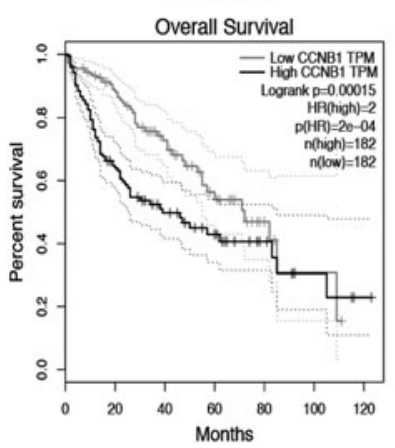

CDK1

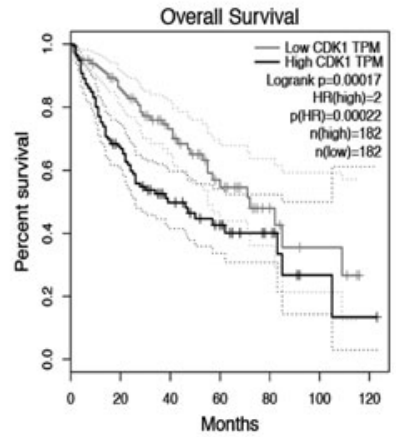

BIRC5

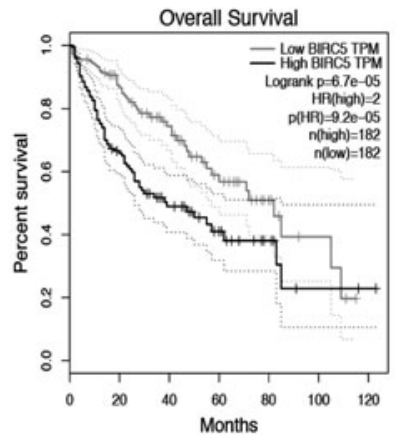

CDCA5

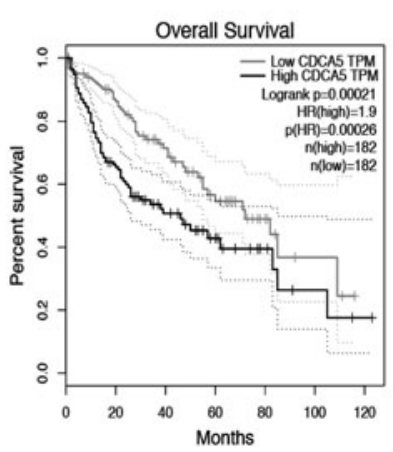

IGF2

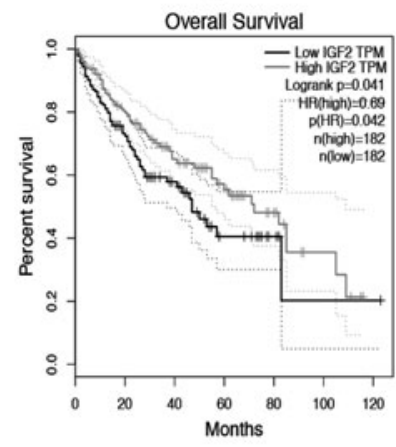

CENPF

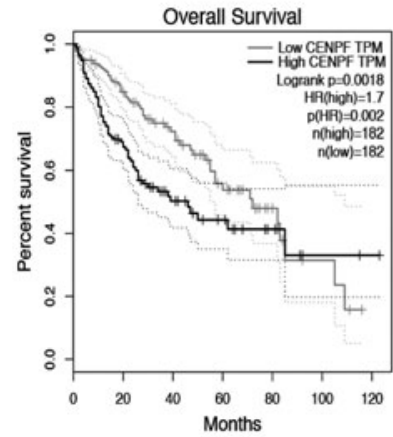

NCAPG
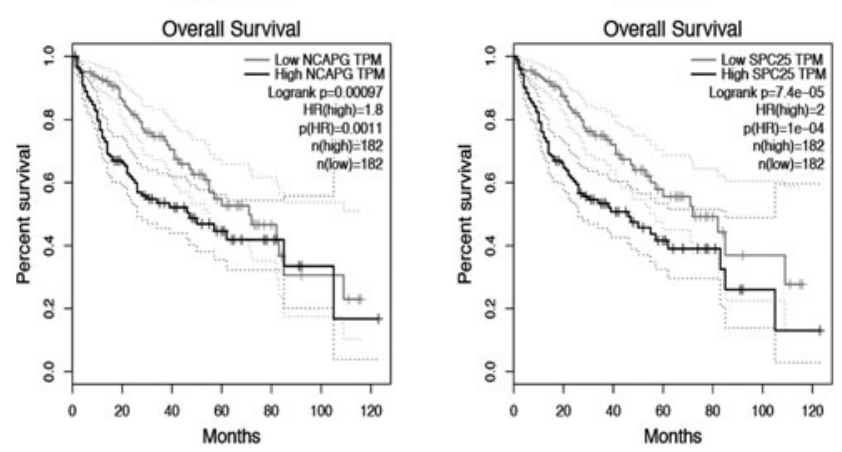

CDCA8

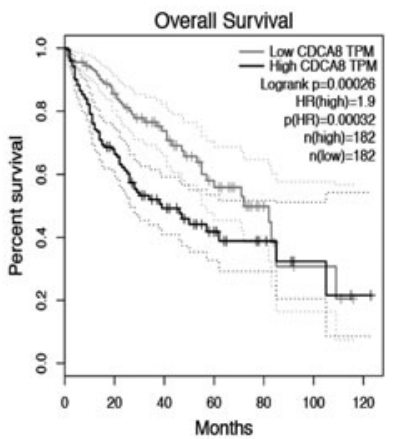

SPC25

CENPU

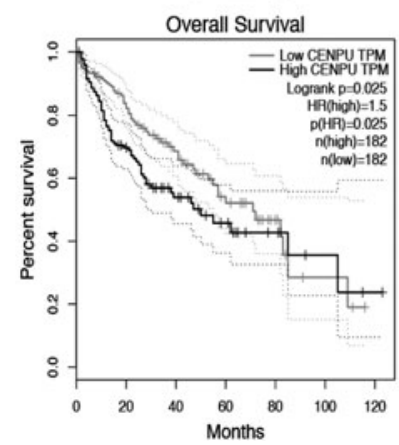

FIG. 3. Overall survival of the genes with expression in accordance with that in TCGA/GTEx datasets. (A) Overall survival of downregulated genes. (B) Overall survival of upregulated genes. TCGA/GTEx, The Cancer Genome Atlas and Genotype-Tissue Expression. 


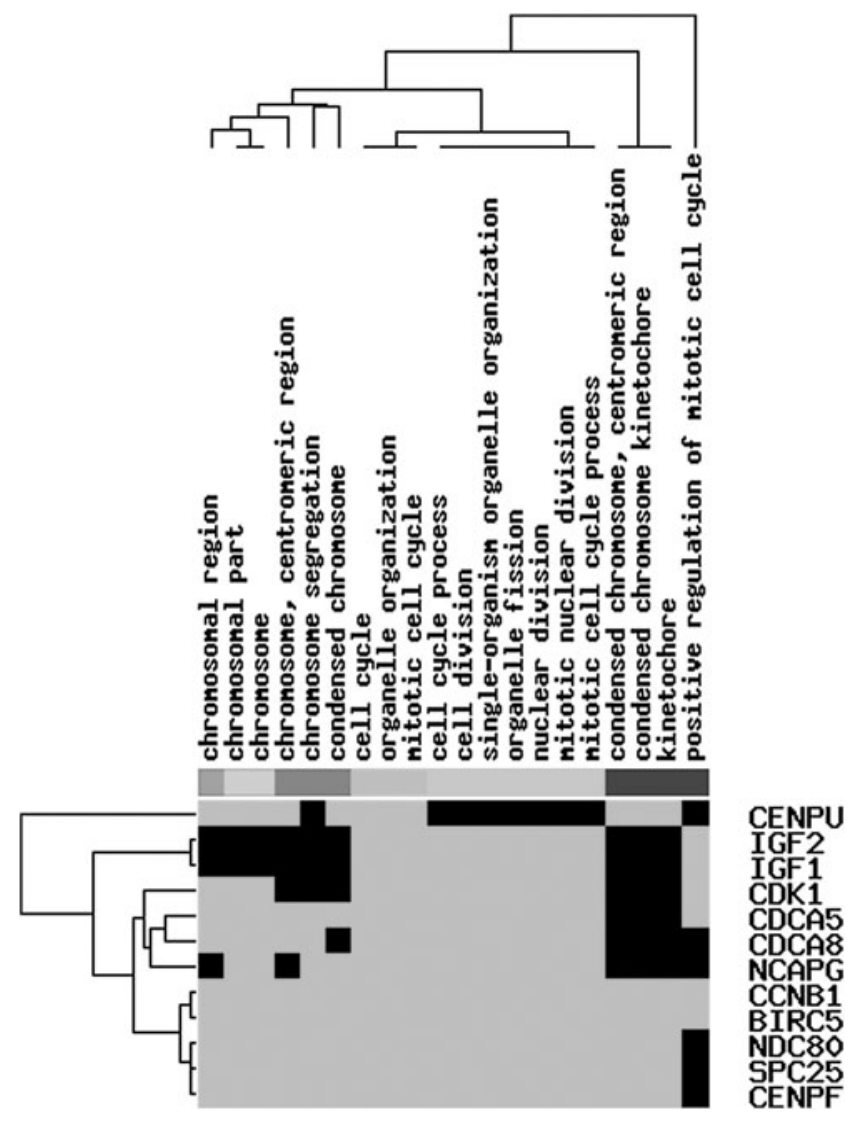

FIG. 4. The functional enrichment analysis of 12 candidate hepatocellular carcinoma-related genes (gray indicates that the gene is enriched in the item, black indicates that the gene is not enriched in the item).

HCC patients. In addition to $I G F 1$, the insulin-like growth factor 2 (IGF2) expression level is also related to the HCC stage (Adamek and Kasprzak, 2018). The nuclear division cycle 80 (NDC80) gene encodes one type of outer kinetochore protein. It forms a heterotetramer with the proteins NUF2, SPC25, and SPC24. This protein complex forms microtubule-binding domains (D'Archivio and Wickstead, 2017). Ju et al. (2017) showed that NDC80 contributes to progression of HCC by reducing apoptosis and overcoming cell cycle arrest. Cyclin-dependent kinase $1(C D K 1)$ is a small protein that is highly conserved, which when coupled with Sox2, is closely related to tumorigenesis in human melanoma (Morgan, 2006; Ravindran Menon et al., 2018). Centromere protein $\mathrm{F}(C E N P F)$ is a protein encoded by the CENPF gene in humans (Zhu et al., 1995; Chan et al., 1998; Li et al., 1998). Studies have shown that coexpression of FOXM1 and CENPF is a robust prognostic indicator of poor survival and metastasis in human prostate cancer (Aytes et al., 2014). Hayama et al.'s research implied that phosphorylation and activation of the cell division cycleassociated $8(C D C A 8)$ protein by aurora kinase B play an important role in human lung tumorigenesis (Satoshi et al., 2007). G2/mitotic-specific cyclin-B1 (CCNB1), known as survivin, is a regulatory protein involved in mitosis (Sartor et al., 1992). Baculoviral inhibitor of apoptosis (IAP) repeatcontaining 5 (BIRC5) is a member of the IAP family. BIRC5 is involved in negative regulation of apoptosis. Survivin is known to be highly expressed in most tumor cell types and absent in normal cells, making it a good target for cancer therapy (Pennati et al., 2007, 2008; Mita et al., 2008; Zaffaroni et al., 2010). Non-SMC condensin I complex subunit G $(N C A P G)$ is a protein encoded by the $N C A P G$ gene (Jäger et al., 2000; Kimura et al., 2001; Geiman et al., 2004). The Liu et al. (2018) group suggested that silencing NCAPG inhibits proliferation and induces apoptosis in HCC cells. Spindle pole body component 25 homolog (SPC25), a component of the NDC80 kinetochore complex, plays a crucial role in regulating mitotic chromosome segregation in prostate cancer (Liu et al., 2018). Cell division cycle-associated 5 (CDCA5) serves an important role in promoting colorectal cancer progression by activating the ERK signaling pathway (Shen et al., 2019). Additionally, CDCA5 could be associated with HCC (Tian et al., 2018), breast cancer (Phan et al., 2018), and lung carcinogenesis (Nguyen et al., 2010). Centromere protein $\mathrm{U}(C E N P U)$ promotes nonsmall cell lung cancer proliferation involving the Wnt/ $\beta$-catenin signaling pathway and predicts poor survival through the expression of FOXM1 (Wang et al., 2018; Zhang et al., 2018).

In conclusion, in this study, we have identified 12 potential candidate HCC-related genes, most of which have previously been implicated in multiple pathways associated with tumorigenesis. In addition, we identified two novel genes, CENPU and SPC25, as potential markers of HCC that have not been previously implicated in carcinogenesis. All of these DEG candidate HCC-related genes should be confirmed through molecular biological experiments.

\section{Authors' Contributions}

All authors participated in the data analysis. Bin Zhao and Zheng Wan performed the comparative analysis using bioinformatic tools. Bin Zhao interpreted the data and wrote the manuscript. All authors read and approved the final manuscript.

\section{Author Disclosure Statement}

The authors declare no potential conflicts of interest with respect to the research, authorship, and/or publication of this article.

\section{Funding Information}

No funding was received for this article.

\section{Supplementary Material}

Supplementary Figure S1

\section{References}

Adamek A, Kasprzak A (2018) Insulin-Like Growth Factor (IGF) System in Liver Diseases. Int J Mol Sci 19:1308.

Ashburner M, Ball CA, Blake JA (2000) Gene ontology: tool for the unification of biology. The Gene Ontology Consortium. Nat Genet 25:25-29.

Aytes A, Mitrofanova A, Lefebvre C, et al. (2014) Crossspecies regulatory network analysis identifies a synergistic interaction between FOXM1 and CENPF that drives prostate cancer malignancy. Cancer Cell 25:638-651.

Balogh J, Victor D 3rd, Asham EH, et al. (2016) Hepatocellular carcinoma: a review. J Hepatocell Carcinoma 3:41-53. 
Barrett T, Troup DB, Wilhite SE, et al. (2009) NCBI GEO: archive for high-throughput functional genomic data. Nucleic Acids Res 37(Suppl_1):D885-D890.

Benjamini Y, Hochberg Y (1995) Controlling the false discovery rate: a practical and powerful approach to multiple testing. J R Statist Soc B (Methodological) 57:289300.

Chan GK, Schaar BT, Yen TJ (1998) Characterization of the kinetochore binding domain of CENP-E reveals interactions with the kinetochore proteins CENP-F and hBUBR1. J Cell Biol 143:49-63.

Chang YS, Adnane J, Trail PA, et al. (2007) Sorafenib (BAY 43-9006) inhibits tumor growth and vascularization and induces tumor apoptosis and hypoxia in RCC xenograft models. Cancer Chemother Pharmacol 59:561-574.

Chen DS, Sung JL, Sheu JC, et al. (1984) Serum alphafetoprotein in the early stage of human hepatocellular carcinoma. Gastroenterology 86:1404-1409.

D'Archivio S, Wickstead B (2017) Trypanosome outer kinetochore proteins suggest conservation of chromosome segregation machinery across eukaryotes. J Cell Biol 216:379-391.

Dennis G Jr., Sherman BT, Hosack DA, et al. (2003) DAVID: Database for annotation, visualization, and integrated discovery. Genome Biol 4:R60.

Dessau RB, Pipper CB (2008) "R"-project for statistical computing. Ugeskrift Laeger 170:328-330.

Edgar R, Domrachev M, Lash AE (2008) Gene Expression Omnibus. Nucleic Acids Res 30:207-210.

Franceschini A, Szklarczyk D, Frankild S, et al. (2013) STRING v9.1: protein-protein interaction networks, with increased coverage and integration. Nucleic Acids Res 41 (Database issue):D808-D815.

Geiman TM, Sankpal UT, Robertson AK, et al. (2004) Isolation and characterization of a novel DNA methyltransferase complex linking DNMT3B with components of the mitotic chromosome condensation machinery. Nucleic Acids Res 32:2716.

Guo Y, Bao Y, Ma M, Yang W (2017) Identification of Key Candidate Genes and Pathways in Colorectal Cancer by Integrated Bioinformatical Analysis. Int J Mol Sci 18:722.

Hayama S, Daigo Y, Yamabuki T, et al. (2007) Phosphorylation and activation of cell division cycle associated 8 by aurora kinase B plays a significant role in human lung carcinogenesis. Cancer Res 67:4113-4122.

Jäger D, Stockert E, Jäger E, et al. (2000) Serological cloning of a melanocyte rab guanosine 5'-triphosphate-binding protein and a chromosome condensation protein from a melanoma complementary DNA library. Cancer Res 60:3584-3591.

Ju LL, Chen L, Ji JH (2017) Effect of NDC80 in human hepatocellular carcinoma. World J Gastroenterol 23:3675-3683.

Keating GM (2008) Mecasermin. BioDrugs 22:177-188.

Kimura K, Cuvier O, Hirano T (2001) Chromosome condensation by a human condensin complex in Xenopus egg extracts. J Biol Chem 276:5417-5420.

Kohl M, Wiese S, Warscheid B (2011) Cytoscape: software for visualization and analysis of biological networks. Data mining in proteomics, Humana Press 696:291-303.

Korpelainen E, Tuimala J (2014) RNA-Seq Data Analysis: A Practical Approach. Chapman and Hall (CRC Press), Boca Raton.

Lee HJ, Lau BT, Ji HP (2013) Targeted Sequencing Strategies in Cancer Research. In: Wu W, Choudhry H (eds.), Next Generation Sequeuncing in Cancer Research: Volume 1: Decoding the Cancer Genome. Springer Science+Business Media, New York. pp.137-159.
Li S, Ku CY, Farmer AA, et al. (1998) Identification of a novel cytoplasmic protein that specifically binds to nuclear localization signal motifs. J Biol Chem 273:6183-6189.

Liu K, Li Y, Yu B, et al. (2018) Silencing non-SMC chromosome-associated polypeptide $\mathrm{G}$ inhibits proliferation and induces apoptosis in hepatocellular carcinoma cells. Can J Physiol Pharmacol 96:1246-1254.

Llovet JM, Ricci S, Mazzaferro V, et al. (2008) Sorafenib in Advanced Hepatocellular Carcinoma. N Eng J Med 359:378390.

Mita AC, Mita MM, Nawrocki ST, Giles FJ (2008) Survivin: key regulator of mitosis and apoptosis and novel target for cancer therapeutics. Clin Cancer Res 14:5000-5005.

Morgan DO (2006) The Cell Cycle: Principles of Control. New Science Press, New London.

Mou T, Zhu D, Wei X, et al. (2017) Identification and interaction analysis of key genes and microRNAs in hepatocellular carcinoma by bioinformatics analysis. World J Surg Oncol 15:63.

Nguyen MH, Koinuma J, Ueda K, et al. (2010) Phosphorylation and activation of cell division cycle associated 5 by mitogenactivated protein kinase play a crucial role in human lung carcinogenesis. Cancer Res 70:5337.

Ogata H, Goto S, Sato K, et al. (1999) KEGG: Kyoto Encyclopedia of Genes and Genomes. Nucleic Acids Res 27: 29-34.

Pennati M, Folini M, Zaffaroni N (2007) Targeting survivin in cancer therapy: fulfilled promises and open questions. Carcinogenesis 28:1133-1139.

Pennati M, Folini M, Zaffaroni N (2008) Targeting survivin in cancer therapy. Expert Opin Ther Targets 12:463-476.

Phan NN, Wang CY, Li KL, et al. (2018) Distinct expression of CDCA3, CDCA5, and CDCA8 leads to shorter relapse free survival in breast cancer patient. Oncotarget 9:6977-6992.

Ravindran Menon D, Luo Y, Arcaroli JJ, et al. (2018) CDK1 interacts with Sox2 and promotes tumor initiation in human melanoma. Cancer Res 78:6561-6574.

Sartor H, Ehlert F, Grzeschik KH, et al. (1992) Assignment of two human cell cycle genes, $\mathrm{CDC} 25 \mathrm{C}$ and CCNB1, to $5 \mathrm{q} 31$ and 5q12, respectively. Genomics 13:911-912.

Satoshi HD, Yataro Y, Takumi H, et al. (2007) Phosphorylation and activation of cell division cycle associated 8 by aurora kinase B plays a significant role in human lung carcinogenesis. Cancer Research 67:4113-4122.

Shen A, Liu L, Chen H, et al. (2019) Cell division cycle associated 5 promotes colorectal cancer progression by activating the ERK signaling pathway. Oncogenesis 8:19.

Smoot ME, Ono K, Ruscheinski J, et al. (2011) Cytoscape 2.8: new features for data integration and network visualization. Bioinformatics 27:431-432.

Smyth GK (2005) limma: Linear Models for Microarray Data. In: Gentleman R, Carey VJ, Huber W, et al. (eds). Bioinformatics and Computational Biology Solutions Using R and Bioconductor. Statistics for Biology and Health. Springer, New York.

Smyth GK, Speed T (2003) Normalization of cDNA microarray data. Methods 31:265-273.

Tang Z, Li C, Kang B, et al. (2017) GEPIA: a web server for cancer and normal gene expression profiling and interactive analyses. Nucleic Acids Res 45(W1):W98-W102.

Thurnherr T, Mah WC, Lei Z, et al. (2016) Differentially Expressed miRNAs in Hepatocellular Carcinoma Target Genes in the Genetic Information Processing and Metabolism Pathways. Sci Rep 6:20065. 
Tian Y, Wu J, Chagas C, et al. (2018) CDCA5 overexpression is an Indicator of poor prognosis in patients with hepatocellular carcinoma (HCC). BMC Cancer 18:1187.

Wang J, Li YC, Deng M, et al. (2017) Serum insulin-like growth factor-1 and its binding protein 3 as prognostic factors for the incidence, progression, and outcome of hepatocellular carcinoma: a systematic review and meta-analysis. Oncotarget 8:81098.

Wang JH, Zhao LF, Lin P, et al. (2014) GenCLiP 2.0: a web server for functional clustering of genes and construction of molecular networks based on free terms. Bioinformatics 30: 2534-2536.

Wang SY, Feng LY, Meng ZQ (2015) Bicluster and pathway enrichment analysis related to tumor progression of hepatocellular carcinoma. Eur Rev Med Pharmacol Sci 19:11911197.

Wang X, Chen D, Gao J, et al. (2018) Centromere protein U expression promotes non-small-cell lung cancer cell proliferation through FOXM1 and predicts poor survival. Cancer Manag Res 10:6971-6984.

Wilhelm SM, Carter C, Tang L, et al. (2004) BAY 43-9006 exhibits broad spectrum oral antitumor activity and targets the RAF/MEK/ERK pathway and receptor tyrosine kinases involved in tumor progression and angiogenesis. Cancer Res 64:7099-7109.
Zaffaroni N, Pennati M, Daidone MG (2010) Survivin as a target for new anticancer interventions. J Cell Mol Med 9: 360-372.

Zhang Q, Li YD, Zhang SX, Shi YY (2018) Centromere protein $\mathrm{U}$ promotes cell proliferation, migration and invasion involving $\mathrm{Wnt} / \beta$-catenin signaling pathway in nonsmall cell lung cancer. Eur Rev Med Pharmacol Sci 22: 7768-7777.

Zhao B, Baloch Z, Ma Y, et al. (2019) Identification of potential key genes and pathways in early-onset colorectal cancer through bioinformatics analysis. Cancer Control 26:1-8.

Zhu X, Mancini MA, Chang KH, et al. (1995) Characterization of a novel 350-kilodalton nuclear phosphoprotein that is specifically involved in mitotic-phase progression. Mol Cell Biol 15:5017-5029.

Address correspondence to:

Bin Zhao, MD, PhD

School of Medicine

Xiamen University

Xiamen 361000

China

E-mail: zhaobinxmu@163.com 